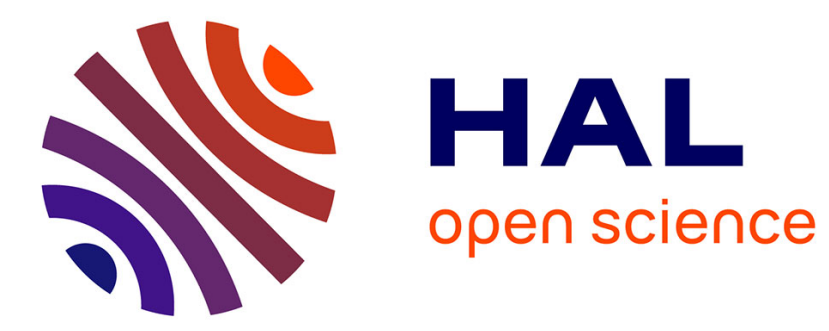

\title{
A Systematic Review on the Use of Groupware Technologies in Emergency Management
}

Jacimar F. Tavares, Leandro Da Silva Gonçalves, Patrícia Gonçalves, Marcos Borges

\section{- To cite this version:}

Jacimar F. Tavares, Leandro Da Silva Gonçalves, Patrícia Gonçalves, Marcos Borges. A Systematic Review on the Use of Groupware Technologies in Emergency Management. 3rd International Conference on Information Technology in Disaster Risk Reduction (ITDRR), Sep 2018, Poznan, Poland. pp.22-35, 10.1007/978-3-030-32169-7_3 . hal-02799293

\section{HAL Id: hal-02799293 \\ https://hal.inria.fr/hal-02799293}

Submitted on 8 Jun 2020

HAL is a multi-disciplinary open access archive for the deposit and dissemination of scientific research documents, whether they are published or not. The documents may come from teaching and research institutions in France or abroad, or from public or private research centers.
L'archive ouverte pluridisciplinaire HAL, est destinée au dépôt et à la diffusion de documents scientifiques de niveau recherche, publiés ou non, émanant des établissements d'enseignement et de recherche français ou étrangers, des laboratoires publics ou privés.

\section{(c)(1)}

Distributed under a Creative Commons Attribution| 4.0 International License 


\title{
A Systematic Review on the Use of Groupware Technologies in Emergency Management
}

\author{
Jacimar F. Tavares, Leandro da Silva Gonçalves, \\ Patrícia do Amaral Gurgel M. Gonçalves and Marcos R.S. Borges \\ Programa de Pós-Graduação em Informática, Instituto de Matemática, Universidade Federal do \\ Rio de Janeiro, Rio de Janeiro, Brazil \\ \{jacimar.tavares, lsg.nce, patriciaagm\}@gmail.com, \\ mborgeseppgi.ufrj.br
}

\begin{abstract}
The term groupware has been used in the context of emergencies aimed at, among other things, allowing the management and execution of emergencies to be carried out by more than one involved, at the same time, cooperating and/or coordinating. This work aims to analyze the state of the art about groupware technologies created by researchers to assist in the management of emergencies. In this sense, the technologies and their areas of action were surveyed and analyzed: pre-emergency event (preparation and mitigation, where planning and preparation for a possible emergency occur; during the emergency (whose objective is to promote the execution of actions and follow); and post emergency event, which comprises the phase of restructuring, reconstruction and regeneration, for example, of the sites hit by the emergency. The results of the systematic literature review show that in the last years there has been more effort in the design of groupware technologies that work in pre and post-emergence to detriment of the post-emergency phase, opening up the possibility for developers of groupware technologies to consider exploiting such phase.
\end{abstract}

Keywords: Groupware, Emergency management, Systematic literature review.

\section{$1 \quad$ Introduction}

The term groupware has been used in the context of emergencies aimed at, among other things, allowing the management and execution of emergencies to be carried out by more than one involved, at the same time, cooperating and/or coordinating [1].

Groupwork, in the context of using cooperative learning techniques and using computational technologies, has proposals that, in the practice of group activities, students have the opportunity to learn about processes that are for groups, in which refers to their personal strategies (relative to that of other people), relying on different perspectives on the same subject [2].

When the subject is emergency management there are those who confuse the term with emergency response, the first one includes other issues that go beyond action after an emergency occurs, as it includes risk mitigation in order to prevent or reduce the impact (such as building levees or removing people from flood-prone locations, disaster 
preparedness and disaster preparedness, disaster response actions (such as search and rescue), and recovery of, for example, basic services to the population. The area of emergency management itself has evolved from the 1940s to today, becoming multiorganizational, intergovernmental and intersectoral collaborative [3], comprising the phases: (i) pre-event (Preparation and Mitigation), at which stage planning and preparation for a possible emergency event occurs, where the objective is to increase the probability of an emergency response being successful; (ii) during the event (Response), the stage at which the emergency event occurs. A response is needed to save lives, material assets, evacuation of victims, reduction of risks (or their total elimination), provision of food, water, shelter and medical assistance or recovery of essential public services, and (iii) phase where reconstruction, restructuring, regeneration, rehabilitation and replenishment take place in order to maintain the resilience of the affected site, in addition to being characterized as an opportunity to improve previous aspects of the place reached by the event [26].

This work supports the idea that new work conducted by researchers that aims to develop groupware technologies to support emergency management, however, need to consider existing technologies and published in the literature in order to provide new contributions to the community. In this sense, it presents a Systematic Review of Literature (SRL) [4] to verify the state of the art on existing publications, with the purpose of verifying the distribution of groupware technologies developed in the respective phases of the emergency.

Among the reasons for a systematic literature review are the need to synthesize evidence in the literature about, for example, some technology or empirical evidence of the benefits and limitations of an agile method. Also, it can be used to identify possible gaps in current research in order to suggest new areas for future research. Another possibility would be the need to provide a framework to adequately position new research activities [4].

In order to carry out a systematic literature review, it is necessary to perform the following steps, namely [5]: (i) Systematic Review Planning, which defines the actions that will be taken and the steps to be followed, as well as to be investigated. All this becomes feasible through the definition of an SRL protocol, which aims to allow a formal search for tools, following well defined criteria, unlike an ad-hoc research approach that does not have a judicious methodology for obtaining results through research [5]; (ii) The accomplishment of the revision, step in which the actions planned in the protocol are executed; (iii) Identification and selection of research, which seeks to search for publications that will be analyzed in the context of SRL; (iv) Evaluation of the quality of the study selected; (v) Extraction and monitoring of the data to be obtained, defined in the protocol; (vi) Synthesis of the analyzed data and (vii) Communication of the review, where the final records are made and the dissemination of what was obtained.

In this work it was possible to identify different systems that were created or are being studied to provide important data to adequately support decision making, because in this complex environment there are several challenges due to the dynamics of unpredictable events [23]. However much of it still focuses on the static or limited scope approach, managing sensor data or geographic mapping of a region. 
Due to the growth of Information and Communication Technologies, there are studies that show the opportunity to explore the most dynamic aspect of data collection through the organized cross-referencing of social media information with existing information and its use in a more collaborative way, as it brings the spontaneous participation of citizens, especially those who live or work near the occurrence of events [25]. Such crossbreeding provides an increase in the quality of information used both in monitoring and in responding to an emergency situation. Social media users can be instrumental in assisting teams during planning and their specialties can be discussed in a collaborative way to achieve a better outcome in both their actions and in response to emergencies.

Another important point observed in this RS, are the tools and the adherence of the same the phases of the emergency. Considering the following phases: pre-emergency event, during emergency event (response) and after emergency event, it was possible to observe that few are the tools that meet the 3 phases of the emergency. Most of them attend the phase during the emergency event (response).

During the analysis of the work, it was possible to observe the effort to use data from existing tools, popular as social networks or systems already existing in the organizations that work in the emergency area. Thus creating new solutions from these data and interfaces. Tools that use social networking data $[12,16]$ and tools that integrate embedded tool data [9] show intent and effort to search existing solutions as a data source.

This article has focused on tools of the last 3 years, and despite the growth and popularization of social networks, we can see that there is in this respect, there is a gap for the organization of such data. The use of two tools was used as social networking software.

The subsequent phases: pre-emergency event, during the emergency event (response) and post emergency event, were possible so that the few are the tools that meet the 3 phases of the emergency. Most respond to a phase during the emergency event (response).

This paper is divided as follows: section 2 presents the systematic review planning, exposing the protocol that was developed and used in SRL. Section 3 presents the steps of the execution of the SRL and the results obtained and section 4 the final considerations.

\section{Planning the Systematic Review of Literature}

This study presents the planning of SRL led assembly the protocol of systematic literature review and the elements that has it, such as issues of research and search of string.

\section{Protocol of the Systematic Review of Literature}

The protocol defined in this SRL is composed of (i) goal of SRL; (ii) criteria for acceptance and rejection of publications; (iii) research questions; (iv) search string; (v) definition of the databases of publications; and (vi) a methodology, as part of the planning of the SRL, which will guide the execution of the SRL. 
The goal of this SRL is to analyze the state-of-the-art literature that presents groupware technologies that have been developed or are used in emergency management. Therefore, it is intended to raise all of the groupware tools and describe how each one of them supports the management of emergencies.

The criterion for acceptance of publications is that it focuses on the presentation of some software tool that is characterized as groupware for emergency management and that has been implemented or presented or analyzed within the scope of the research in which the article was generated, in the last 3 years.

The criteria for rejection of publications are (i) that it does not contain the implementation or presentation or analysis of any software tool that is characterized as groupware for emergency management within the scope of the research in which the publication was generated; or (ii) that refers to the management of emergencies in the context of medicine (publications of this nature may describe, among other things, strategies in surgeries, which is beyond the scope of this SRL); or (iii) publications that only present conceptual frameworks, studies or analyzes of emergency databases; or (iv) publications that are not in the range of the last 3 years.

The Research Questions that are intended to be addressed in this SRL are questions that should be answered at the end of this work. The issues are:

- Q1: What is the production of the community, from the bases surveyed, in the last 10,5 and 3 years?

- Q2: What groupware tools have been developed or are being used to support emergency management in the last 3 years?

- Q3: Among the stages of emergency management (pre-emergency, emergency and post-emergency) in the last 3 years, which have had less contributions in terms of developed groupware technology?

- Q4: Data from social networks, or from existing systems are being exploited and used in groupware systems?

The Q3 research question is the principal question to be answered in this systematic review, since it will allow researchers, with an interest in groupware, to have a vision of which steps there has been less contribution to the development of groupware.

The search string was formulated with the intention of allowing the execution of queries, in the SRL Execution stage, in bibliographic databases aiming the retrieval of publications that are appropriate to the context of this work:

- ((computer-supported AND collaborative AND work) OR (collaborative AND work) OR (collaborative AND approach) OR (cscw) OR (cscw AND based) OR (collaboration)) AND ((crisis AND management) OR (disasters AND management) OR (emergency AND response) OR (crisis AND informatics)) AND (groupware OR system OR systems OR tool OR tools OR plugin OR software).

In the context of this SRL, the following publications databases will be used to enable the execution of the created search string. Five electronic databases of publications with free access for students of the Federal University of Rio de Janeiro (UFRJ) were considered: 
- El Compendex (http://www.engineeringvillage.com)

- IEEE Digital Library (http://ieeexplore.ieee.org)

- ISI Web of Science (www.isiknowledge.com)

- Scopus (http://www.scopus.com)

The methodology used to obtain publications followed the steps described below:

The websites of the databases used in this SRL have been accessed; As an advanced search, the search string created has been executed; From the publications listed, the bibtex file (format and file extension that has structured information of the articles) was exported. The information extracted from each publication are title, year, authors and abstract. A bibtex file was exported to each of the databases; A tool was used to import the bibtex files. Through it it was possible to analyze the data extracted from each publication; The total of publications by database was counted and the duplicates were eliminated; The total of the last 10 years (2009 to 2018) was then counted; of the last 5 years (2014 to 2018); of the last 3 years (2016 to 2018); From the publications of the last 3 years, the title was analyzed and, according to the defined exclusion criterion, those that were clearly recovered but not the scope of this systematic review were rejected.

From the remaining publications after the title analysis, the abstract was analyzed, respecting the inclusion criteria, in order to eliminate those that clearly showed no evidence that they could be accepted.

Of the remaining publications (i.e. those that could not be eliminated by title and abstract), the quantities were counted per year (2016, 2017 and 2018).

The remaining publications (that is, those that could not be deleted by title and abstract) were analyzed in the context of this systematic review in order to answer the defined research questions. The publications that fit the criteria of acceptance of this SRL were counted and accepted to be presented in this SRL.

In this section the protocol of the systematic literature review was defined. From it, it will be possible to move to the next step, which is the execution of SRL.

\section{Systematic Literature Review Execution}

After the definition of the protocol, a systematic review was performed in order to answer the defined research questions.

\subsection{Execution of database searches}

The results were recorded in the month of May, 2018. The results obtained account for the recovery of the total of 4248 publications, the oldest being retrieved from 1985 and the new ones from the year 2018 until the date of execution of this review. Figure 1 shows the summary of the number of publications retrieved in each of the publications databases. The number of duplicate publications, in the universe of a total of 4248 , is 191, which were eliminated soon after the accounting. Compendex presents the largest 
number of articles (2004). The Scopus, IEEE and Web of Science databases present 779,707 and 567 , respectively.

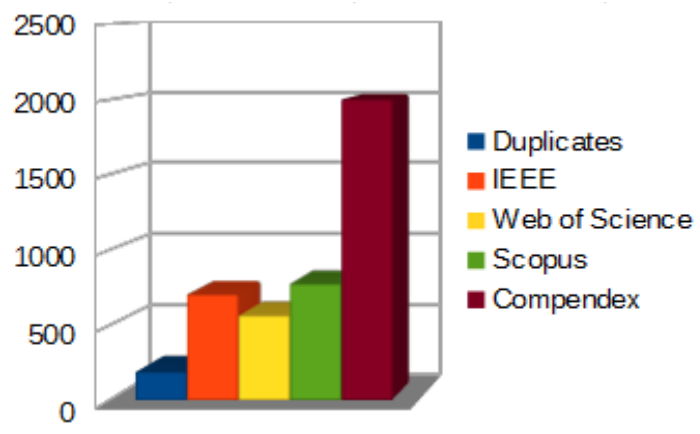

Fig. 1. Summary by Database

\subsection{Analyzes and Results of Research Questions}

After the retrieval of the publications in all bases, the accounting was carried out to answer the first question of research:

- Q1: What is the production of the community, from the bases surveyed, in the last 10,5 and 3 years?

It is considered Figure 2. It presents a summary of the articles distributed in the recovered years.

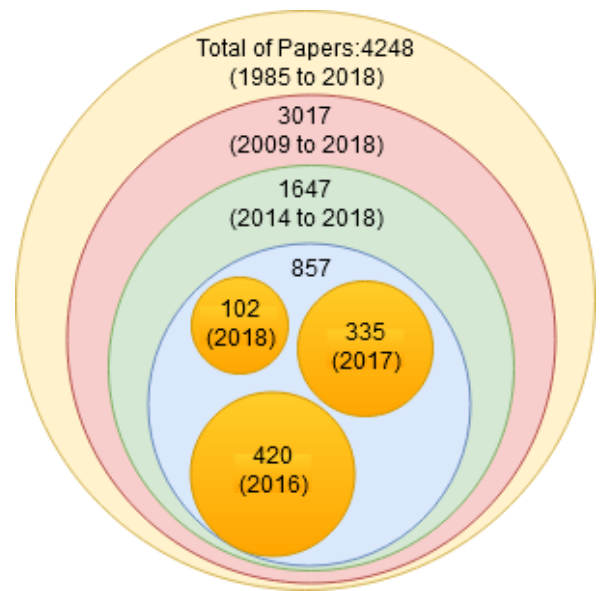

Fig. 2. Summary of publications per year

Of the total of articles recovered it is noticed that the range of publications is of 34 years (from 1985 to 2018). In the last 10 years, the number of articles is 3017 and in 
the last 5 years the quantity falls to 1647 . The last 3 years, which are the publications targeted by this article, count 857, of which 420 were published in 2016, 335 in 2017 and 102 in 2018, to date.

Another research question to be answered is Q2:

- Q2: What groupware tools have been developed or are being used to support emergency management in the last 3 years?

The first step in answering the research question was to perform the analysis of the 857 publications of the last 3 years, starting with the title, then by the abstract, as defined in the protocol methodology. Figure 3 shows the result of the analysis.

The 857 publications of the last 3 years are divided as follows: 102 in the year 2018, 335 in the year 2017 and 420 in the year 2016. These publications have moved on to the next stage, which includes the analysis of the title. Publications were eliminated because they clearly fit the criteria for rejecting publications. At the end of the analysis by title, there were 35 publications of the year 2018, 109 of 2017 and 189 of 2016. Subsequently, the analysis by abstract where the publications that could not be eliminated by the criterion of rejection (for showing signs, in their abstracts that could present groupware tools for emergency management). It is important to highlight that the publications resulting from the abstract analysis stage can, as they are read, demonstrate not having the tools they mention have in their abstracts. As can be seen in Figure 3, 66 publications resulted from the SRL implementation stage, considering the inclusion and rejection criteria, for the last 3 years.

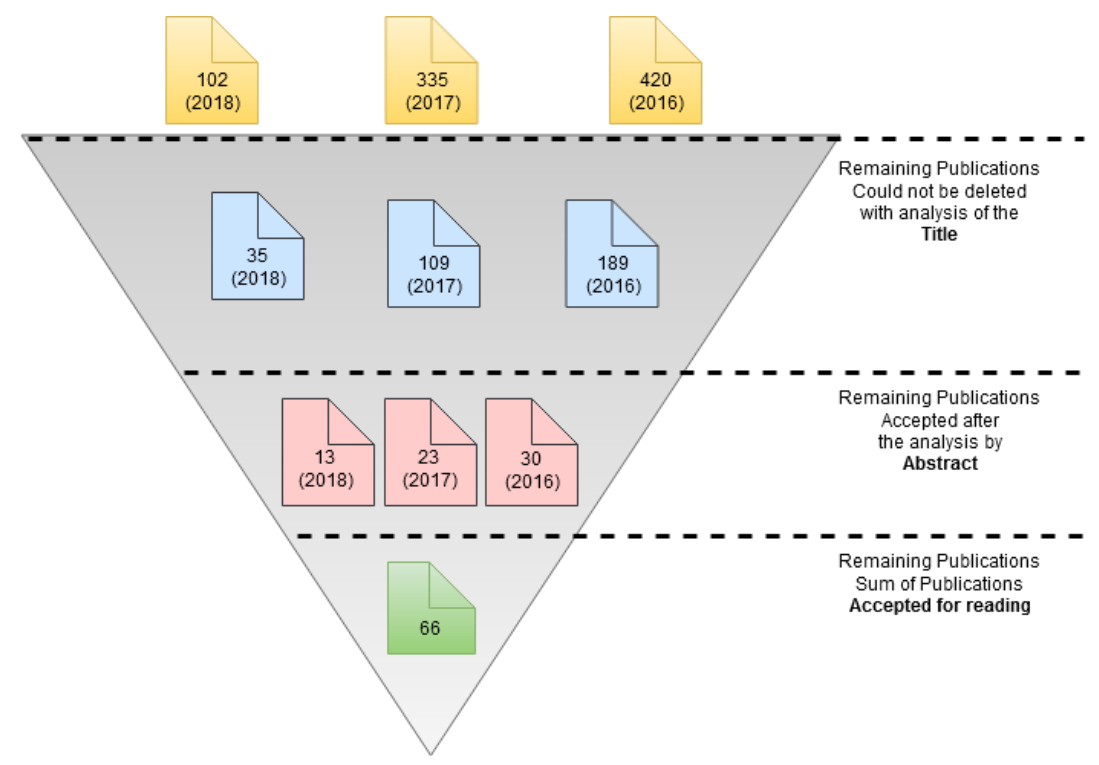

Fig. 3. Summary of publications accepted for review 
After reading and analyzing the 66 articles resulting from the implementation of the SRL, a list was generated that presents a summary of software tools that are characterized as groupware and that support emergency management according to the defined inclusion criterion. These tools were extracted from the 11 publications ([6] to [16]) described below:

Emergency Panel [6] is a system that provides selection of a desired data set and presents them using a configurable panel. The system focuses on intuitive user interaction, flexibility and collaboration. It also seeks to reduce the effects of information overload and hence improve the quality of decisions.

SituMap and PhotoSorter [7] are discussed in relation to their potential contribution to each of the phases of the crisis life cycle model (pre-crisis, crisis and post-crisis). Both are social media applications for groupware and are designed to facilitate communication between first responders and crisis teams during a crisis. These are applications that collaborate to improve risk identification, crisis response, and communication processes throughout the crisis lifecycle.

Panacea Cloud [8] incorporates a new dashboard that provides augmented reality benefits with minimal human communication through the integration of an Incident Command System (ICS) standardized with Internet of Things (IoT), as virtual headsup (HUDs) beacons, QR-code cards, and wireless mesh network elements.

The Panacea Cloud enables First Aid to simultaneously assist patients while communicating with others and the Incident Commander in a disaster scenario where local communication with traditional systems is affected and / or unavailable.

EPISECC [9] Common Information Space (CIS) - It is an adapter to share and provide collaborative information on emergency care across multiple systems and institutions. It seeks to provide integration between existing systems without the need to develop a single system for everyone involved.

ClickOnMap [10] Platform that enables the configuration of systems that collect and make available VGI Voluntary Geographic Information (VGI, which obtains information through user-generated content) in a short period of time, and can be used by the administration to interact with the citizen. By collecting VGI you can capture data and generate information that is difficult to obtain automatically such as place names, exposure of infrastructure problems, natural post-disaster mapping, real-time firefighting logging, or population data from a region. Therefore, such information is made available to emergency management teams providing better planning and execution of emergency response actions.

Crowdtasking [11] is a centralized form of crowdsourcing for crisis and disaster management. In this tool it is possible to have registered volunteers (with their characteristics and profiles), which are triggered as necessary by a coordinator. The dynamics of the tool are as follows: from an incident, the coordinator creates the event, filters the volunteers and triggers the volunteers within the profile. Available volunteers respond to the call and receive the assignments. In conclusion, they send a feedback which will be received by the coordinator. The coordinator evaluates the feedback and generates reports.

EPIC Project [12] Tool designed to scan social networking data to obtain information for emergency response. EPIC Project provides: large-scale data navigation, 
filtering, sharing and visualization for crisis information search. The EPIC Collect architecture consists of the EPIC Collect service that connects to Twitter and retrieves the tweets based on the event information that is managed by the EPIC Event Editor.

The Intelligent Cooperative Transport System (C-ITS) [13] is a system that aims to reduce the response time in emergency situations in traffic accidents, increasing the chances of surviving the injured, through the appropriate coordination of stakeholder collaborations, use of distributed intelligence and automation of the rescue process.

The Disaster Management and Disaster Management System (AYDES) [14], developed on the basis of Turkey's National Disaster Response Plan, makes disaster management and emergencies more efficient and effective, providing better support for decision support. AYDES seeks risk reduction and better preparedness in response and disaster recovery cases.

AYDES operates with several other systems, services and applications (desktop, mobile and web) using Geographic Information Systems (GIS) and Remote Sensing (RS) technologies, which are responsible for providing more useful information for better real-time mapping.

In this review, we present a preliminary work on the Augmented Reality (AR) functionality implemented in the Intelligent Distributed Holistic Emergency Management System (THEMIS) [15], which supports real-time decision makers at command posts and field teams. THEMIS gathers information from various sources (users, sensors, crowdsourcing), coordinates them and disseminates them among its users.

The GIS-based web platform [16] captures a variety of sources, such as voluntary geographic information (VGI), social networks, collaborative projects, physical sensors and official maps, integrating them and making them available through APIs.

The diversity of information sources for the platform assists in the ability to analyze the location of the disaster, its surroundings in real time. This allows for better preparation even before the arrival of the authorities.

The article platform differs from other similar proposals because it can collect data from any other VGI project that provides an API, allowing search by location rather than requiring a dedicated application to collect the data.

- Q3:Among the stages of emergency management (pre-emergency, emergency and post-emergency) in the last 3 years, which have had less contributions in terms of developed groupware technology?

In order to answer it, we tried to classify the tools found in SR, in which phases of the emergency they would be adequate: pre-emergency event, during emergency event (response) and after emergency event.

From the classification on Figure 4 it can be seen that most technologies support emergency management during the incident, which is the response stage ( 9 in total). The post-event stage was characterized by the fact that it is the one with the lowest production of the community, in the analyzed years, however, it can not be said that the area is deficient in developed technologies, since this systematic review was based on the analysis of the publications of a limited set of databases over a specific set of years and did not consider, for example, whether the publications of other years than those 
analyzed have already filled the possible gaps in the implementation of groupware technologies. It is also important to highlight that some tools have the proposal of acting in more than one phase, as is the case of the tools SituMap and PhotoSorter, Project EPIC and ClickOnMap.

\begin{tabular}{|l|c|c|c|c|}
\hline \multicolumn{1}{|c|}{ TOOL } & $\begin{array}{c}\text { PRE- } \\
\text { EVENT }\end{array}$ & $\begin{array}{c}\text { DURING } \\
\text { THE EVENT }\end{array}$ & $\begin{array}{c}\text { POST } \\
\text { EVENT }\end{array}$ & YEAR \\
\hline Emergency Dashboard & & $\mathrm{X}$ & & 2016 \\
\hline SituMap e PhotoSorther & $\mathrm{X}$ & $\mathrm{X}$ & $\mathrm{X}$ & 2016 \\
\hline Nuvem Panacéia & & $\mathrm{X}$ & & 2016 \\
\hline EPISECC & $\mathrm{X}$ & $\mathrm{X}$ & & 2017 \\
\hline ClickOnMap & $\mathrm{X}$ & $\mathrm{X}$ & & 2017 \\
\hline Crowdtasking & & $\mathrm{X}$ & & 2017 \\
\hline Projeto EPIC & $\mathrm{X}$ & $\mathrm{X}$ & & 2017 \\
\hline C-ITS & $\mathrm{X}$ & $\mathrm{X}$ & $\mathrm{X}$ & 2018 \\
\hline THEMIS & $\mathrm{X}$ & $\mathrm{X}$ & & 2018 \\
\hline AYDES & & & & 2018 \\
\hline GIS & 6 & 9 & 2 & 11 \\
\hline TOTAIS & & & & 2018 \\
\hline
\end{tabular}

Fig. 4. Presents the groupware technologies categorized by the stages in which an emergency event can occur.

- Q4: Data from social networks, or from existing systems are being exploited and used in groupware systems?

We have the analysis of the Q4 research question. Data from social networks or existing systems are exploited and used in groupware system. Of the 11 papers analyzed, two of them were created from data generated through social media $[12,16]$ and one of them was based on existing data in legacy systems [9], aiming at the reuse of data and exploration of popular tools, in the case of social networks, in order to provide groupware tools adhering to users' daily lives.

Of the 55 publications that could not be accepted by the inclusion criterion after reading, (although they were not rejected after reading title and abstract), the graph of Figure 5 was generated, which shows a overview of what in fact they were to publications.

Among the publications, it was possible to perceive 13 studies, such as [17], which aims to explore themes that result from the content generated by user posts on the Facebook page of an emergency management agency. The study shows that Facebook has been used primarily for information dissemination, coordination and collaboration, information search, identity building and social provisions in the context of emergency management. In publishing, most of the fundamental principles of the national disaster recovery framework were met using Facebook's data on emergency management. So, it is highlighted as social media, in this case Facebook can be a good source of information and content to support emergency management. 
Of the 5 publications that presented services, it can be highlighted [18]. Based on SOA, it aims to collect and standardize the data by providing integration between various agencies, which use different systems. The proposed approach aims to improve the interoperability of data sharing between different agencies and support decision-making for flood management.

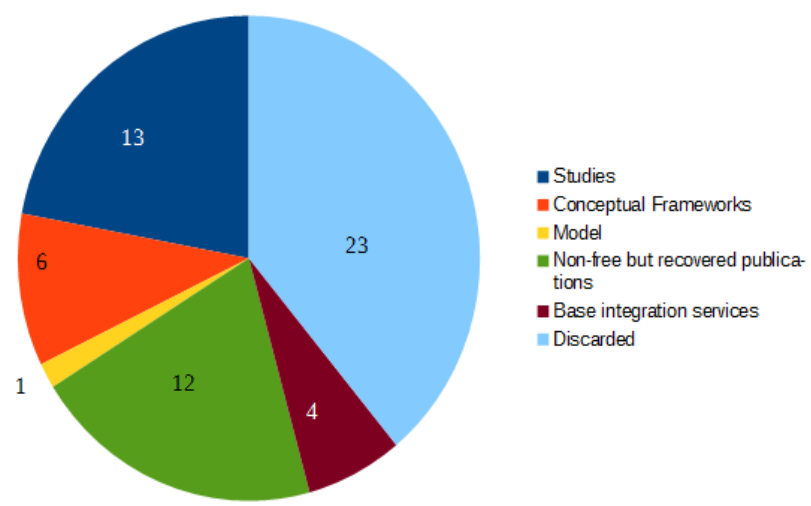

Fig. 5. Overview of rejected publications

The probable flood event information can be used to issue alerts in addition to planning for future acquisition and data collection as a service for flood simulation (using hydrological models), flood mapping (using automated procedures), damage assessment, rescue and operations. It can help planning and decision making for information on flood management. The purpose of the paper is to design a SOA-driven, event-driven flood management mechanism.

In the 7 publications that approach conceptual frameworks, without implementations, it is possible to highlight the one presented in [19], where the need for a platform that unifies information from several collaborative sources and supports the actions of first responders in the emergency is raised. Currently, libraries are capable of representing disasters and related information, but are not able to map them, either static or dynamically, because they do not have specific symbols for it, the various areas of expertise and responsibilities of the cartographers involved must be considered. Appropriate mapping of computer-assisted disaster situations is necessary for the timely representation and sharing of disaster information, response situations and decisions to support management and mitigate the risks of new disasters. This will also allow you to align status information with emergency or transsexual emergency teams that are already at the scene and in most cases perform the first response actions, generating new information from the location [24].

The other publications correspond to models (total of 1); non-free publications which, despite being retrieved in consultation with public access libraries and with free publications, were not available for download or reading (total 5); and others discarded, because they only presented theories or did not present the implemented tools (in some cases, only specified), despite the fact that they could not have been eliminated in the title and abstract analysis. 


\subsection{Possible Research in Collaborative Work in Emergency Management}

In the past, lack of data was one of the main barriers faced by public managers in the decision-making process, however, today the situation has been reversed [22].

One of the biggest concerns in monitoring and planning actions in emergency situations is optimizing all the resources involved (time, personnel, material and others) to obtain the best possible result from all those involved (people or organizations), since the higher the level of preparedness, based on reliable information, the greater the likelihood of a successful outcome, that is, appropriate emergency treatment with the least possible impact on the victims involved, or without occurrence of casualties.

In order to achieve this desired result, different Information Systems have already been created or are being studied to provide important data for decision making, but much of it still focuses on the static approach or limited scope, managing sensor data or geographic mapping of a region.

Due to the advancement of Information and Communication Technologies, there are studies that show the opportunity to explore the most dynamic aspect of data collection, through the organized cross-referencing of social media information with the existing information and its use in a more collaborative Such crossbreeding increases the quality of the information used both in monitoring and in responding to an emergency situation. People can be local eyes and ears who will assist the teams during their planning and their specialties can be discussed collaboratively in order to achieve a better result both in their actions and in the actions of emergency response.

The need to communicate and distribute information during the emergency event is fundamental to the care process. For Kapucu [20] it is highlighted that from an analysis of the attack on the Twin Towers that the distribution and coordination of information is of great importance. From interviews with the participants of the ransom in the 9/11 crisis, [21] report that technology and communication were key and distributions are still a major problem in the response to the crisis. Therefore, communication studies are critical to understanding human behavior and seeking improvements and technologies that support organizations in emergency situations for communication, sharing and collaboration.

With the support of publications of people in a particular region where there is an occurrence to be attended, it is possible that the emergency team is adequately prepared to provide care; use better routes because you will know more traffic behavior in the face of the emergency; move equipment and people necessary to carry out their actions; help the possible victims in the most efficient way possible, since it will have in hand the necessary material and the planned hospital logistics.

The collaboration helps the team in creating a collective knowledge and in real time during service; allows better dissemination of the perceptions of each specialist present; hospitals that receive victims may have remote assistance from specialist physicians for appropriate treatment through interaction with a local physician (telemedicine).

Due to the fact of the popularization of social media it is suggested, as future work, studies on how to use social media as tools or as a source of data and information to support the decision making of the emergency and / or command and control teams. 


\section{Conclusions}

It is important to emphasize that the threats to the validity of the systematic review of literature carried through questions such as the retrieval of publications. In practice, it means that the publications that were analyzed underwent an initial title and abstract filtering. Titles or abstracts that did not indicate that the publications might contain what was expected in terms of acceptance criteria may not have been retrieved. In the same way that abstracts can induce the reader to believe that there are tools implemented in the context of the work, the same cannot be confirmed by reading the article. If this can occur, the opposite can also happen. There may be publications that do not reveal in their titles or abstracts that presented, in their texts, what is sought in this systematic review. If this occurs, they will not be considered for reading because they do not present information that induce such verification.

Another point is that this systematic literature review is not intended to retrieve all publications that may fit into the acceptance criteria because it was performed considering a small number of databases for publications. Other databases that have not been addressed in this SRL may have been used to index groupware publications in emergency management.

\section{References}

1. Pimentel, M., Fucks, H.: Sistemas Colaborativos. Rio de Janeiro. Elsevier, (2011).

2. Campos, F. C. A, Santoro, F. M, Borges, M. R. S.: Cooperação e aprendizagem on-line. Rio de Janeiro, DP\&A (2003).

3. Waugh, W., Streib, G.: Collaboration and Leadership for Effective Emergency Management. Public Administration Review (2006).

4. Kitchenham, B.A., Charters, S.: Guidelines for performing systematic literature reviews in software engineering. Tech. Rep. EBSE 01, Keele University, Keele, UK (2007).

5. Kitchenham, B.: Procedures for performing systematic reviews. Technical Report, TR/SE 0401, Department of Computer Science, Keele University, Keele, UK (2004).

6. Nascimento, B. S., Vivacqua, A. S., Borges, M. R. S.: A flexible architecture for selection and visualization of information in emergency situations. IEEE International Conference on Systems, Man, and Cybernetics (SMC), (2016).

7. Maresh-Fuehrer, M. M., Smith, R.: Social media mapping innovations for crisis prevention, response, and evaluation. Journal Computers in Human Behavior 54(C), 620-629 (2016).

8. Gillis, J., Calyam, P., Apperson, O., Ahmad, S.: Panacea's Cloud: Augmented reality for mass casualty disaster incident triage and co-ordination. 13th IEEE Annual Conference on Consumer Communications \& Networking Conference (CCNC), (2016).

9. Neubauer G. et al.: Common information space as enabler for collaboration in disaster management: Demonstration of the validity of the EPISECC CIS concept. International Conference on Information and Communication Technologies for Disaster Management (2017).

10. Câmara, J. H. S. et al.: OnMap: A platform for development of Volunteered Geographic Information systems. Iberian Conference on Information Systems and Technologies (2017)

11. Auferbauer, D., Telliolu, H.: Centralized crowdsourcing in disaster management: Findings and implications. In: Proceeding of the 8th International Conference on Communities , pp. 173-182 (2017). 
12. Barrenechea, M. et al.: Getting the query right for crisis informatics design issues for webbased analysis environments. Journal of Web Engineering . 16 (5+6), 399-432 (2017).

13. Chen, L., Englund, C.: Every Second Counts: Integrating Edge Computing and Service Oriented Architecture for Automatic Emergency Management. Journal of Advanced Transportation (2018).

14. Keskin, I. et al.: Geographic information system and remote sensing based disaster management and decision support platform: AYDES, International Archives of the Photogrammetry, Remote Sensing and Spatial Information Sciences - ISPRS Archives (2018).

15. Nunes, I. L., Lucas, R., Simoes-Marques, M., Correia, N.: Augmented Reality in Support of Disaster Response. Advances in Intelligent Systems and Computing (2018).

16. Fonte, C. C., Fontes, D., Cardoso, A.: A web GIS-based platform to harvest georeferenced data from social networks: Examples of data collection regarding disaster events. International Journal of Online Engineering (2018).

17. Kurian, J. C., John, B. M.: User-generated content on the Facebook page of an emergency management agency: A thematic analysis. Emerald Publishing Limited.

18. Sharma, V. K. et al.: Event-driven flood management: design and computational modules. Geo-spatial Information Science 19 (1), 39-55.

19. Peng, G. et al.: Construction of collaborative mapping engine for dynamic disaster and emergency response. Natural Hazards (2018).

20. Kapucu, N.: Interagency Communication Networks During Emergencies: Boundary Spanners in Multiagency Coordination. American Review of Public Administration 36 (2) 207-225 (2006).

21. Dawes, S.S., Cresswell, A.M., Cahan, B.B.: Learning from Crisis: Lessons in Human an Information Infrastructure from the World Trade Center Response. Social Science Computer Review 22, 52-66 (2004).

22. Marino, T., Campos, M. L. M, Borges, M. R. S., Breslin, J.: Architecture for gathering and integrating collaborative information for decision support in emergency situations. International Journal of Information and Communication Technology 12, 345-363 (2018).

23. Cordeiro, K. F., Campos, M. L. M., Borges, M. R.S.: aDApTA: Adaptive approach to information integration in dynamic environments. Computers in Industry 71, 88-102 (2015).

24. Freitas, D. P., Borges, M. R. S.,Carvalho, P. V. R., Vivacqua, A.S.: Communication channels among various roles during crisis response. In: Proceeding of XIII International Conference on Information Systems for Crisis Response and Management, 2016, Rio de Janeiro.

25. Freitas, D. P., Borges, M. R. S.,Carvalho, P. V. R.,: A Framework for Dealing Collaborativelywith Interactions from Social Media in Emergency Situations. In: Proceeding on IEEEInternational Conference on Computer Supported Cooperative Workin Design, 2017, Wellington, Nova Zelandia.

26. Rubim, I. C. ; Borges, M. R.S.: The Resilience and Its Dimensions. In: Proceeding of XIV International Conference on Information Systems for Crisis Response and Management (ISCRAM), vol. 1, pp. 457-463, Albi, France (2017). 